\title{
Late Stages of Ribosome Assembly Revealed by Cryo-Electron Microscopy
}

\author{
A. Jomaa ${ }^{1}$, G. Stewart ${ }^{1}$, J. Martín-Benito ${ }^{2}$, R. Zielke $^{3}$, T. L. Campbell ${ }^{1}$, J. R. Maddock ${ }^{3}$, E D. \\ Brown $^{1}$, Joaquin Ortega ${ }^{1}$
}

${ }^{1}$ Department of Biochemistry and Biomedical Sciences and MG. DeGroote Institute for Infectious Diseases Research, McMaster University, 1200 Main Street West, Hamilton, Ontario, L8N3Z5, Canada; ${ }^{2}$ Centro Nacional de Biotecnología (CSIC), Campus de Cantoblanco, 28049 Madrid, Spain; ${ }^{3}$ Department of Molecular, Cellular and Developmental Biology, University of Michigan, Ann Arbor, Michigan 48109

Four decades after early assembly studies demonstrated that ribosome assembly is a controlled process [1], our understanding of ribosome assembly is still in its infancy. Just as structure determination has been so important to understanding ribosome function [2] so too will it be critical to sorting out the assembly process [3]. However, the fast pace of assembly of ribosomes in bacteria prevents accumulation of assembly intermediates and makes any attempt of structural characterization of the assembly process in vivo unfeasible. Here, we shows an experimental system that causes the accumulation of specific ribosomal subunit intermediates in vivo to allow their structural characterization. The obtained structures provide a three-dimensional view of the ribosome assembly process.

We have made use of a viable deletion in the $y j e Q$ gene in $E$. coli, a putative ribosome assembly factor [4], to isolate and structurally characterize immature $30 \mathrm{~S}$ subunits assembled in vivo. We showed by Northern blot analysis that the 16S rRNA of these small subunits was incompletely processed. Using isotope tagging proteomic methodology, we demonstrated that they also had an incomplete protein complement. Cryo-electron microscopy reconstructions revealed that the presence of precursor sequences in the rRNA induces a severe distortion in the 3' minor domain of the subunit involved in the decoding of mRNA and interaction with the large ribosome subunit (FIG. 1) [5]. This cryo-EM reconstruction constitutes the first structure of an in vivo assemble immature ribosomal subunit.

This study establishes that the YjeQ protein is a bona fide ribosome assembly factor and provides new guiding principles of the ribosomal assembly process, such as that RNA processing events induce local conformations directing the structure toward the mature assembly. We also concluded that rRNA processing, folding and the entry of tertiary r-proteins are interdependent events in the late stages of $30 \mathrm{~S}$ subunit assembly. These findings demonstrate how studies of emerging assembly factors in ribosome biogenesis can help to elucidate the path of subunit assembly in vivo.

\section{References}

1. S. Mizushima et al., Nature 226 (1970) 1214.

2. B. S. Schuwirth et al., Science 310 (2005) 827-834.

3. M.T. Sykes et al., Annu. Rev. Biophys. 38 (2009) 197-215.

4. D.M. Daigle et al. J. Bacteriol. 186 (2004). 1381-1387. 
5. A. Jomaa et al. RNA (2011) in press.
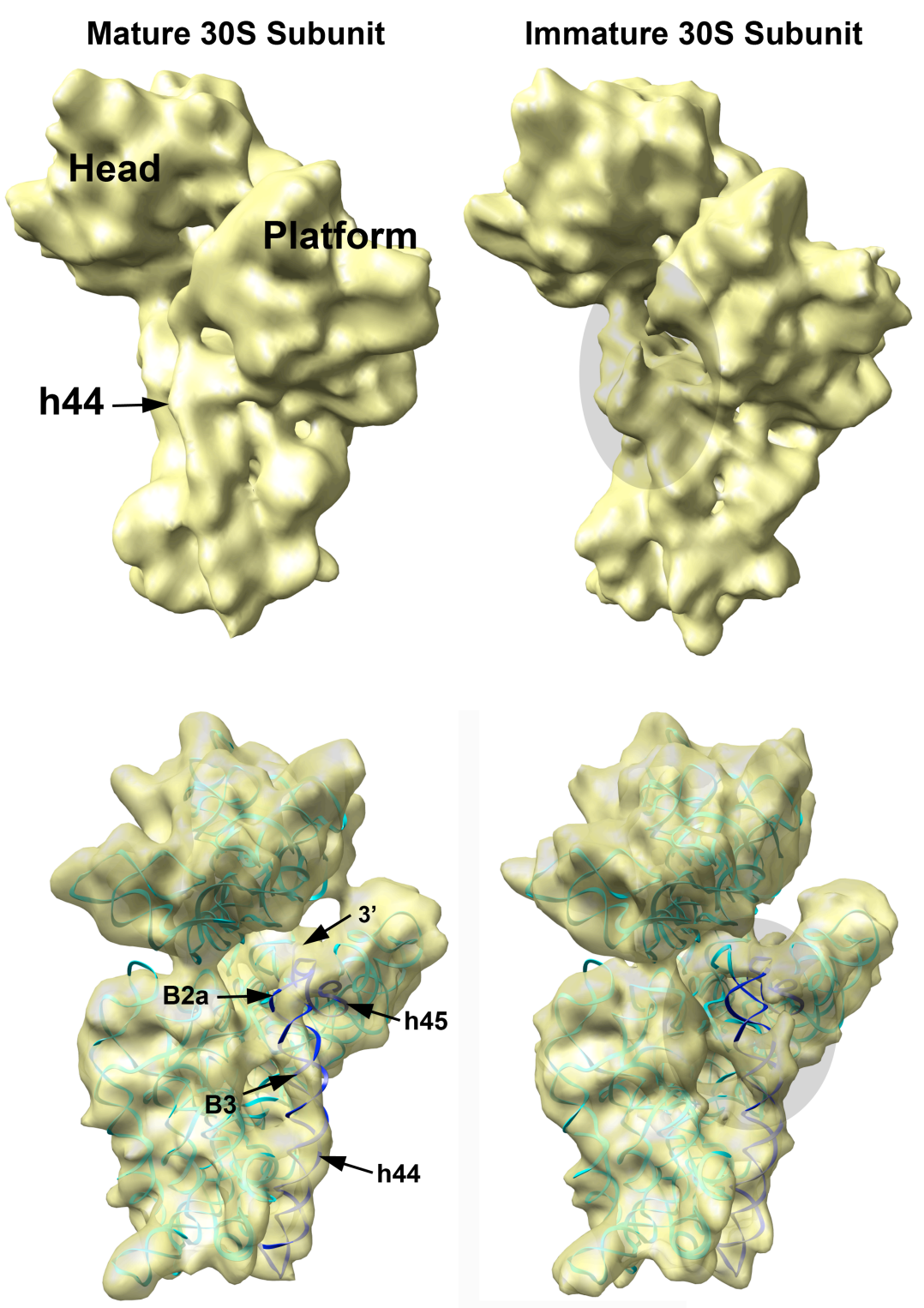

FIG. 1. 3D Reconstruction of the immature 30S ribosomal subunits purified from $\triangle y j e Q$ E.coli cells. Platform-view of the cryo-EM maps of the immature (upper right panel) and mature (upper left panel) $30 \mathrm{~S}$ subunits. Main landmarks of the $30 \mathrm{~S}$ subunit are indicated. In the panels below, the $\mathrm{X}$-ray structure of the wild type $30 \mathrm{~S}$ subunit, shown as a ribbon representation, was fitted into the cryo-EM maps of both the mature (left) and immature (right) $30 \mathrm{~S}$ subunits to illustrate their divergences. In the X-ray structure the rRNA is shown in cyan except helix 44 and 45 that are colored in dark blue. The r-proteins are not shown. Main landmarks of the 30S subunit indicated include helix 44 (h44), helix 45 (h45), the intersubunit bridges B2a (B2a) and B3 (B3) important for the interaction with the 50S subunit and the 3' terminus of the 16S rRNA molecule (3'). The shadowed ovals in the right panels show the area in the helix 44 and decoding center distorted in the EM map of the immature 30 S subunit. 